\title{
Cytomegalovirus Infection of the Central Nervous System Stem Cells from Mouse Embryo: A Model for Developmental Brain Disorders Induced by Cytomegalovirus
}

\author{
Isao Kosugi, Yuichiro Shinmura, Hideya Kawasaki, Yoshifumi Arai, Ren-Yong Li, \\ Satoshi Baba, and Yoshihiro Tsutsui
}

Second Department of Pathology, Hamamatsu University School of Medicine, Hamamatsu, Japan

\begin{abstract}
SUMMARY: Cytomegalovirus (CMV) is the most frequent infectious cause of developmental disorders of the central nervous system (CNS) in humans. Infection of the CNS stem cells seems to be primarily responsible for the generation of the brain abnormalities. In this study, we evaluated the infectivity of murine CMV (MCMV) in epidermal growth factor (EGF)-responsive CNS stem cells prepared from fetal mouse brains, and studied the effect of infection on growth and differentiation of the stem cells. The CNS stem cells were permissive for MCMV infection, although MCMV replication was slower than in mouse embryonic fibroblasts. MCMV infection inhibited the growth and DNA replication of the stem cells. A clonogenic assay revealed that MCMV infection suppressed generation of colonies from single stem cells. When uninfected stem cells were induced to differentiate, a decrease in expression of the primitive neuroepidermal marker nestin was observed by immunocytochemistry and flow cytometry, whereas expression of neurofilament and glial fibrillary acidic protein (GFAP) were induced. In virus-infected CNS stem cells, nestin expression was retained, whereas the expression of neurofilament was more severely inhibited than that of GFAP in these cells. Two-color flow cytometry showed that differentiated glial precursor cells were preferentially susceptible to MCMV infection. MCMV-infected and uninfected CNS stem cells were transplanted into the neonatal rat brains. The reduced number of infected stem cells were engulfed into the subventricular zone and expressed GFAP, but did not migrate further, in contrast to the uninfected stem cells. These results suggest that suppression of the growth of the CNS stem cells and inhibition of the neuronal differentiation by CMV infection may be primary causes of disorders of brain development in congenital CMV infection. (Lab Invest 2000, 80:1373-1383).
\end{abstract}

$C$ ytomegalovirus (CMV), a member of the herpesvirus group, is the most significant infectious cause of congenital anomalies of the central nervous system (CNS) caused by intrauterine infection in humans (Weller, 1971; Ho, 1991), with an average incidence of $1 \%$ of all live births (Stagno et al, 1986; Demmler, 1991). Microcephaly is the most prominent manifestation of the brain abnormalities in congenital CMV infection, with other abnormalities including periventricular calcification, hydrocephalus, cerebellar atrophy, optic nerve atrophy, and polymicrogyria (Becroft, 1981; Bale, 1984; Ho, 1991). However, the exact pathogenesis of these brain abnormalities has not been fully elucidated. Because studies of human subjects have obvious limitations and CMV has strict species specificity, we have developed model sys-

Received April 19, 2000.

This work was supported in part by a grant (9P-4-27) from the National Center of Neurology and Psychiatry of the Ministry of Health and Welfare, Japan, and a grant (10670284) from the Ministry of Education, Science and Culture, Japan.

Address reprint requests to: Dr. Isao Kosugi, Second Department of Pathology, Hamamatsu University School of Medicine, 3600 Handa-cho, Hamamatsu 431-3192, Japan. FAX: 8153435 2224; E-mail: kos180@tm.hama-med.ac.jp. tems for brain abnormalities induced by infection of mouse embryos with murine CMV (MCMV) (Tsutsui et al, 1993; Tsutsui, 1995).

During neurogenesis of the brain, large numbers of neural cells are generated from the CNS stem cells in the ventricular zone facing the ventricular cavity, where self-renewing proliferation of the stem cells occurs, and from which the precursor cells derived from the stem cells migrate, undertaking differentiation at the same time (Davis and Temple, 1994; Chenn and McConnell, 1995; McKay, 1997; Stemple and Mahanthappa, 1997). Because CMV preferentially tends to infect the ventricular and subventricular zones during congenital infection (Becroft, 1981; Perlman and Argyle, 1992), proliferation and differentiation of the stem cells may be affected by the infection. Recently it has been reported that primary multipotent CNS stem cells can be isolated from embryonic and adult brains, and have the capacity to proliferate in response to growth factors such as epidermal growth factor (EGF) and to differentiate into precursor cells expressing neuronal or glial phenotypes (Reynolds and Weiss, 1992, 1996; Vescovi et al, 1999). There has been no report concerning the effects of CMV infection on the biological behavior of the CNS stem cells. In this report, we show that EGF-responsive CNS 
stem cells prepared from fetal mouse brains were permissive for MCMV infection, and that the virus infection suppressed their growth and inhibited their differentiation, especially neuronal differentiation. We transplanted the MCMV-infected and uninfected CNS stem cells into neonatal rat brains to confirm that the effects of MCMV infection on the CNS stem cells in vitro support the phenomena in vivo.

\section{Results}

\section{Infects EGF-Responsive CNS Stem Cells and Inhibits Their Growth}

In the previously established serum-free culture medium containing EGF (Reynolds and Weiss, 1996), a single CNS stem cell (Fig. 1A) proliferated in response to EGF, generated a floating aggregate of stem cells (Fig. 1B), and formed a "sphere" 80-100 $\mu \mathrm{m}$ in size after 5 days in culture (Fig. 1C). Immediately after passage, dissociated stem cells were infected with MCMV at a multiplicity of infection (MOI) of 1 plaque forming unit (PFU) per cell. At 1 day post-infection (dpi) there was no morphological difference between MCMV-infected and uninfected stem cells (Fig. 1, B and D). The cytopathic effect (CPE), which indicates the beginning of lytic cell death, was observed in the spheres infected with MCMV at $5 \mathrm{dpi}$, rendering spheres of irregular shape (Fig. 1E) compared with the symmetrical spheres of uninfected stem cells (Fig. 1C). The time courses of viral titers and viral antigen expression of CNS stem cells were slower than those of similarly infected mouse embryonic fibroblasts (MEF), which are known to be fully permissive cells for MCMV replication (Fig. 2, A and $\mathrm{B}$ ). The viral titers produced by stem cells peaked at $5 \mathrm{dpi}$ (Fig. 2A). By 3 dpi, more than $90 \%$ of MCMV-infected stem cells showed nuclear fluorescence detected by the N2 monoclonal antibody specific for the MCMV major immediate early (IE)-89K-antigen (Shinmura et al, 1997a) (Figs. 1F and 2B). Growth arrest or decrease in cell number was observed in the infected stem cells, whereas uninfected stem cells grew exponentially. The inhibition of growth was more marked as the MOI was increased (Fig. 2C). The transient decrease in cell number observed at $1 \mathrm{dpi}$ was thought to be caused by the mechanical dissociation of spheres during passage, as described by Reynolds and Weiss (1996). These results indicated that mouse EGF-responsive CNS stem cells were permissive for MCMV replication in vitro, although their permissiveness was lower than that of MEF, and that infection resulted in cell growth inhibition.

\section{Infection Inhibits the DNA Synthesis of CNS Stem Cells}

We tried to determine whether the growth inhibition of MCMV-infected CNS stem cells is caused by the inhibition of cell cycle progression, as observed in human fibroblasts infected with human cytomegalovirus (HCMV) (Lu and Shenk, 1996; Dittmer and Mocarski, 1997). A bromodeoxyuridine (BrdU) incorporation assay was used to examine the effect of MCMV infection on the DNA synthesis of CNS stem cells. At 15 hours post-infection (hpi), when viral DNA had not yet begun to accumulate, the labeling index of MCMVinfected CNS stem cells was significantly decreased as the MOI was increased (Fig. 3A). At MOI 10, the
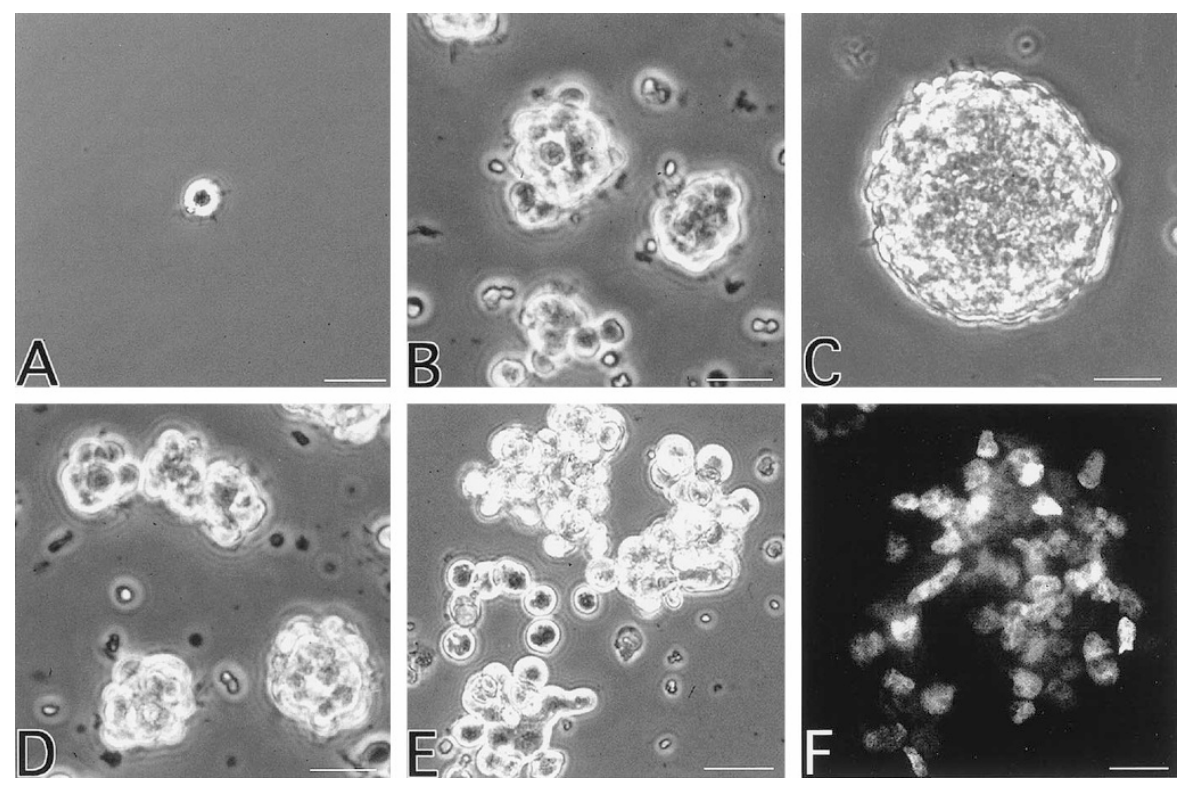

Figure 1.

Phase contrast micrographs of uninfected (A to $C$ ) and murine cytomegalovirus (MCMV)-infected (D and E) epidermal growth factor-responsive central nervous system (CNS) stem cells, and immunofluorescence for MCMV immediate early (IE) antigen (F). A, A single CNS stem cell just after passage. B, Small aggregates of the stem cells 1 day after passage. C, A symmetrical sphere 5 days after passage. D, CNS stem cells infected with MCMV at multiplicity of infection (MOI) 1 , shown at 1 day post-infection (dpi). E, MCMV-infected CNS stem cells showing cytopathic effect on $5 \mathrm{dpi}$. F, MCMV-infected CNS stem cells expressing the IE antigen at 3 dpi. Bar $=20 \mu \mathrm{m}$. 
A

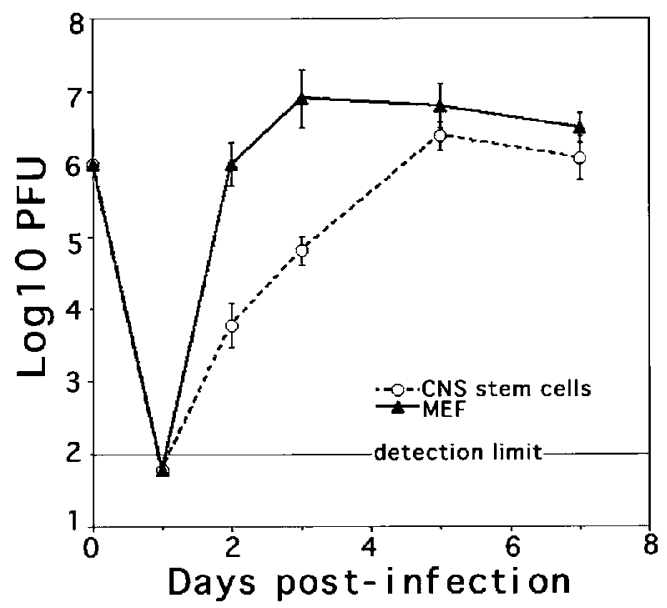

B
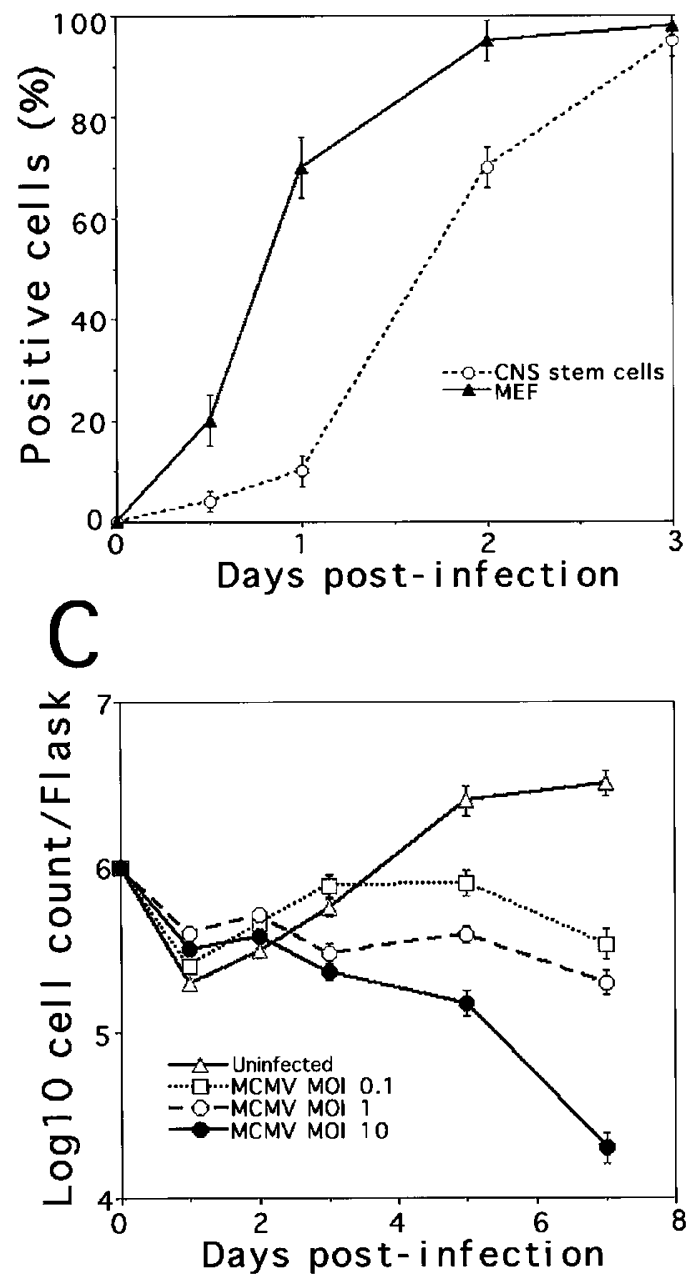

Figure 2.

Time courses of viral titers (A), viral antigen expression (B), and cell growth (C) after MCMV infection of the CNS stem cells or mouse embryonic fibroblasts (MEF). A, MCMV titers in CNS stem cells and MEF infected with MCMV at MOI 1. B, Flow cytometric detection of MCMV IE antigen expression in CNS stem cells and MEF infected with MCMV at MOI 1. C, Growth of CNS stem cells not infected or infected with MCMV at MOI 0.1, 1, and 10. index was reduced to $50 \%$ of that of uninfected stem cells. Two-color flow cytometric analysis clearly demonstrated that the BrdU incorporation was blocked in the cells expressing the IE antigen (Fig. 3B). These data indicate that MCMV infection inhibits the DNA synthesis of the CNS stem cells, and that the blockade of the cell cycle progression occurs during the IE or early phase of infection, but before the start of viral DNA synthesis, similar to HCMV infection of human fibroblasts (Lu and Shenk, 1996; Dittmer and Mocarski, 1997).

\section{Infection Inhibits Self-Renewal of the CNS Stem Cells}

We investigated the effect of MCMV infection on the self-renewal of stem cells in the EGF-responsive spheres; such self-renewal is pivotal for definition of the stem cells and has been tested by the clonogenic assay (Potten and Loeffler, 1990; Reynolds and Weiss, 1996). Seven days after the plating of the dissociated, diluted stem cells, at least $8 \%$ of the uninfected stem cells, which had been plated at approximately 6 cells per well in 96-well plates, had regenerated as spheres (Fig. 4). Each sphere was considered to have been generated from a single stem cell. In MCMV-infected stem cells, the number of regenerated spheres per plate decreased as the $\mathrm{MOI}$ increased. At MOI 10, the number of regenerated spheres was reduced to $18 \%$ of that in uninfected stem cells. These results indicate that MCMV infection inhibits the self-renewal of CNS stem cells. However, even at MOI 10, a few unaffected stem cells (less than $1 \%$ of the total stem cells plated) still generated the spheres.

\section{Infection Inhibits the Migration and Differentiation of CNS Stem Cells}

We next examined the effect of MCMV infection on the differentiation of the CNS stem cells. Four days after passage, spheres were infected with MCMV and then cultured in the same medium containing EGF. At 12 hpi, uninfected or MCMV-infected CNS stem cells were adhering to plates coated with poly-D-lysine and laminin, and were induced to differentiate in the medium lacking EGF but supplemented with $2 \%$ horse serum. Soon after plating, the stem cells began to migrate from the periphery of the spheres and to differentiate. In the uninfected cultures 36 hours after induction of differentiation, heterogeneous cells with flattened cytoplasm and elongated processes extended noticeably from the spheres (asterisk in Fig. 5), which were reduced in size (Fig. 5A). In the MCMVinfected cultures, as the MOI was increased, the proportion of cells showing CPE was increased, whereas the proportion of differentiating cells was decreased (Fig. 5, B to D) and there was less reduction of the size of the spheres. These findings suggested that MCMV infection inhibits the migration and differentiation of CNS stem cells.

Immunocytochemical staining demonstrated that undifferentiated spheres expressed a primitive neuroectodermal marker, nestin (Fig. 6A), but did not express glial 

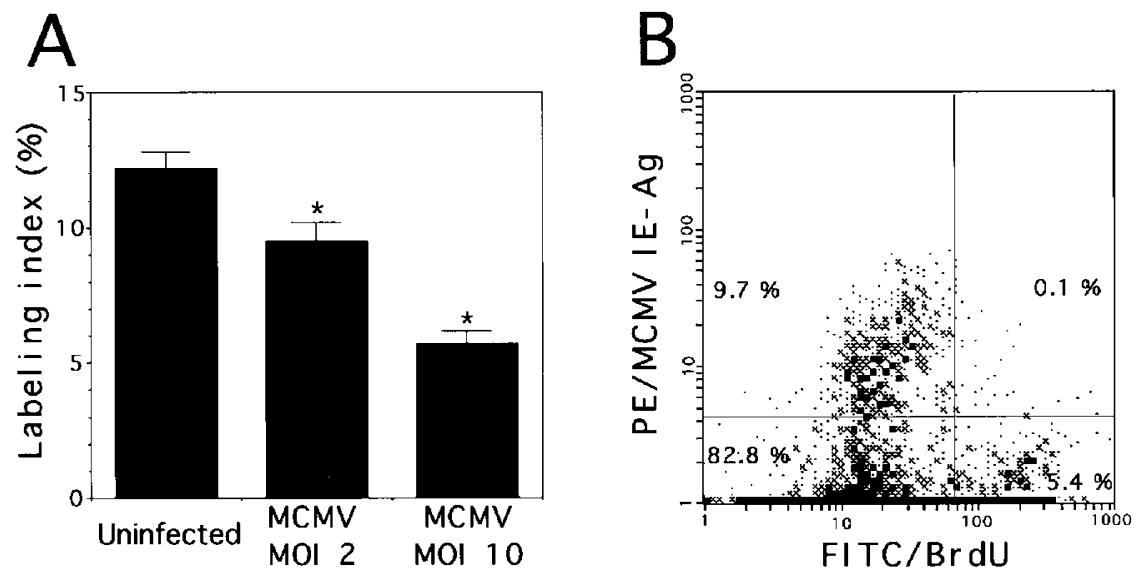

\section{Figure 3.}

Inhibition of DNA synthesis in epidermal growth factor-responsive CNS stem cells by MCMV infection. A, CNS stem cells were not infected or infected with MCMV at MOI 2 or 10. At 15 hours post-infection (hpi), cells were incubated with BrdU for 30 minutes, fixed, stained with anti-BrdU antibody, and analyzed by flow cytometry. Labeling indices were expressed as the average of triplicate determinations. ${ }^{*} p<0.05$ compared with uninfected cells by Student's $t$ test. B, Two-color flow cytometry for BrdU (FITC) and MCMV IE-Ag (PE) in CNS stem cells infected with MCMV at MOI 10. The cut-off points of the relative fluorescence intensity by which positive and negative cells could be conveniently separated were 4.2 or 66.2 , for IE antigen or BrdU, respectively.

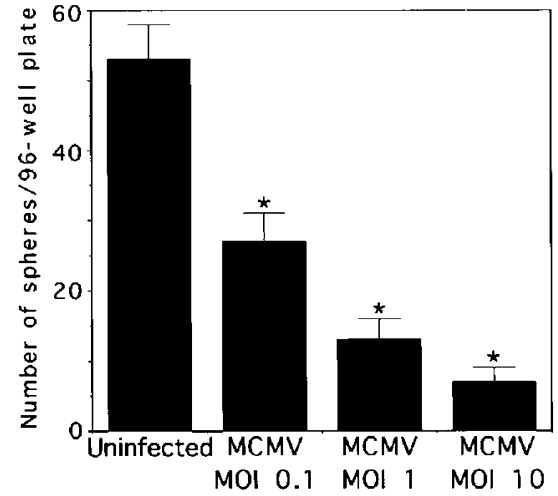

Figure 4.

The effect of MCMV infection on the self-renewal of the CNS stem cells determined by the clonogenic assay. Dissociated CNS stem cells were not infected or infected with MCMV at three different MOI $(0.1,1$, and 10$)$ and plated in 96 -well plates at a density of 6 cells/well. The numbers of regenerated spheres per plate were counted at $7 \mathrm{dpi}$. The averaged data of three independent experiments are shown. ${ }^{*} p<0.05$ compared with uninfected cells in Student's $t$ test.

fibrillary acidic protein (GFAP) (Fig. 6B) or neurofilament (NF) (Fig. 6C). The uninfected cultures 36 hours after differentiation expressed GFAP (Fig. 6E) and NF (Fig. 6F) but still expressed nestin (Fig. 6D). GFAP-positive cells having elongated processes were considered to be glioblasts. NF-positive cells with bipolar shape and fine processes were considered to be neuroblasts (Fig. 4G). Immunocytochemical double staining showed that expression of the IE antigen occurred in nestin-positive (Fig. 6G) and GFAP-positive cells (Fig. 6H), but was scarcely observed in NF-positive cells, which were fewer in number than nestin- and GFAP-positive cells (Fig. 6I).

\section{Infection Inhibited Neuronal Differentiation of CNS Stem Cells More Severely than Glial Differentiation}

The effects of MCMV infection on the expression of the differentiation markers were quantitatively exam- ined by flow cytometry. Expression of nestin was observed in $80 \%$ of undifferentiated stem cells 4 days after passage, whereas less than $1 \%$ of these cells expressed GFAP or NF (Fig. 7Aa). In uninfected cells 36 hours after differentiation, the proportion of nestinpositive cells was reduced to $50 \%$, whereas the proportion of GFAP- and NF-positive cells increased to $34 \%$ and $19 \%$ of the cells, respectively (Fig. 7Ab). The proportion of nestin-positive cells was significantly increased as the MCMV MOI increased, whereas the proportion of GFAP- and NF-positive cells were significantly decreased (Fig. 7, A and B). MCMV infection at MOI 5 reduced the proportions of GFAP- and NF-positive cells to $56 \%$ and $23 \%$ of those in uninfected cultures, respectively (Fig. 7B), suggesting that MCMV infection inhibits the neuronal differentiation of the CNS stem cells more severely than glial differentiation. Two-color flow cytometry demonstrated that NF-positive cells expressing IE antigen at MOI 1 constituted only $8 \%$ of the total IE antigenpositive cells, whereas nestin- or GFAP-positive cells expressing IE antigen constituted $93 \%$ and $51 \%$ of the IE-Ag-positive cells, respectively (Fig. 7C).

\section{Infection Inhibits the Migration of the CNS Stem Cells after Transplantation into Neonatal Rat Brain}

To study the effect of MCMV infection on grafting and in vivo differentiation of the CNS stem cells, we transplanted MCMV-infected and uninfected mouse CNS stem cells into neonatal rat brains, because the developing mouse brain is highly sensitive to MCMV infection, but MCMV does not prominently replicate in the developing rat brain (van den Pol et al, 1999). MCMV-infected and uninfected mouse CNS stem cells detected by the anti-BrdU antibody were observed in the ventricles at 2 days post-transplantation (dpt) (Fig. 8, A and C). The infected stem cells were also stained with the antibody specific to the early nuclear antigen (the D5 antibody) (Fig. 8, E and F). On 

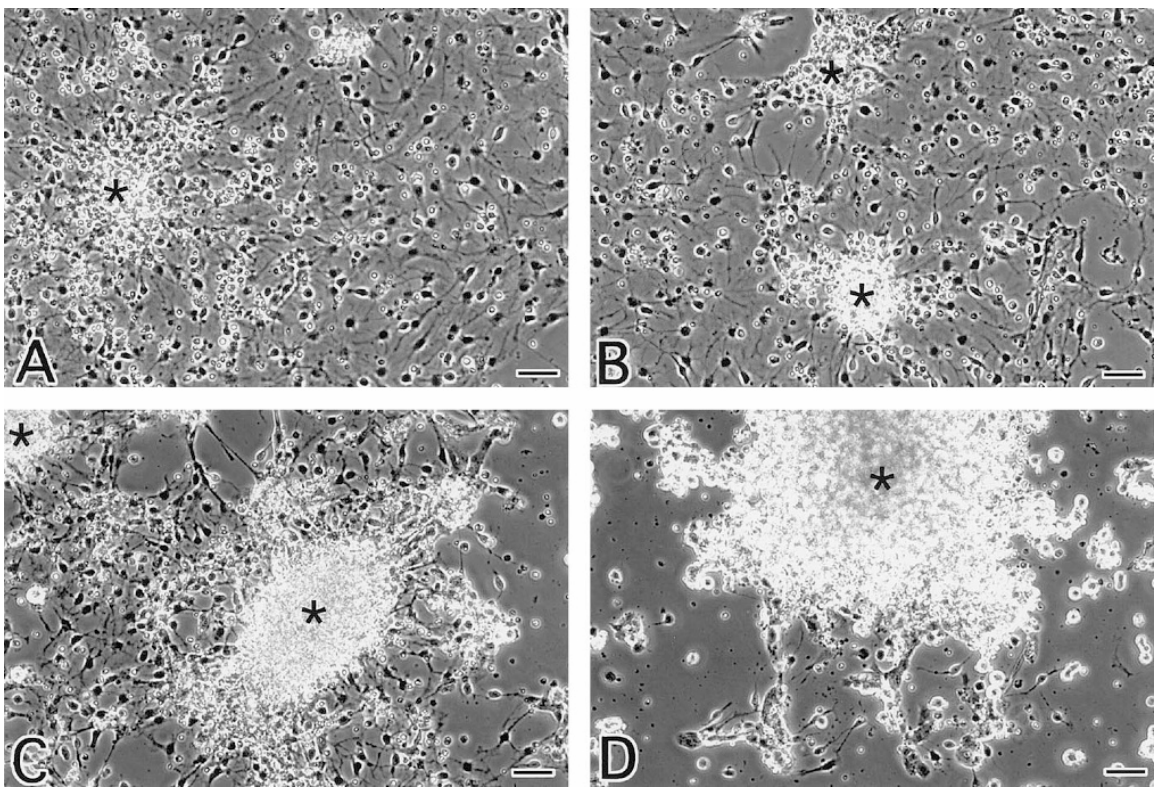

\section{Figure 5 .}

Phase contrast micrographs of the cells differentiated from CNS stem cells 36 hours after induction of differentiation. The spheres of CNS stem cells 4 days after passage were not infected or infected with MCMV at different MOI. At $12 \mathrm{hpi}$, the spheres were allowed to adhere and induced to differentiate in medium lacking EGF but supplemented with $2 \%$ horse serum. A, Uninfected cells. B to D, MCMV-infected cells at MOI 0.2 (B), MOI 1 (C), and MOI 5 (D). * indicates location of the spheres. Bar $=20 \mu \mathrm{m}$.

$6 \mathrm{dpt}$, uninfected stem cells were engulfed into the subventricular zone (SVZ) and further migrated into the white matter (WM) (Fig. 8B), whereas the MCMVinfected CNS stem cells were markedly reduced in number (Fig. 8D), although some were engulfed into the SVZ, but their further migration was hardly detected (Fig. 8D). In the serial adjacent sections, some of the uninfected CNS stem cells in the inner site of the SVZ were found to express GFAP (Fig. 8G), and others in the outer site of the SVZ expressed $\beta 3$-tubulin, an early marker for neuronal differentiation (Tucker, 1990) (Fig. 8H). Expression of GFAP was also found in the grafted infected stem cells in the SVZ (Fig. 8, I and J) on $6 \mathrm{dpt}$, but the expression of the $\beta 3$-tubulin was not detected (data not shown).

\section{Discussion}

In the present study, we showed that the CNS stem cells were susceptible to MCMV replication, and that MCMV infection inhibited the growth and differentiation, especially the neuronal differentiation of the CNS stem cells. These results suggest that infection of the CNS stem cells is primarily responsible for the generation of brain abnormalities in congenital CMV infection in humans. In congenital CMV infection, it is possible that cells in the ventricular zone are primary targets for viral infection, because it has been reported that periependymitis, periventricular necrosis, and calcification are the most frequent findings in the brains of congenital CMV infection (Becroft, 1981; Bale, 1984; Ho, 1991; Perlman and Argyle, 1992). In the ventricular zone, multipotential CNS stem cells have been identified in vitro and in vivo, and shown to be capable of self-renewal and to have the potential to differentiate to neuronal or glial precursor cells in response to some factors (McKay, 1997). The isolated primary CNS stem cells are able to proliferate from single cells to multipotential spherical cell masses in response to EGF (Reynolds and Weiss, 1992, 1996).

We showed that the undifferentiated CNS stem cells were susceptible to MCMV infection, although the viral replication was slower than that in MEF. It has been reported that undifferentiated human embryonal carcinoma (EC) cells were not permissive for HCMV replication, but that some of the cells became permissive when they were induced to differentiate with retinoic acid (Gonczol et al 1984, 1985). The same phenomenon was observed in the mouse EC cells (Dutko and Oldstone, 1981). Similarly, the early mouse embryos were not susceptible to MCMV infection until a certain stage of development (Tsutsui and Naruse, 1987; Kashiwai et al, 1992). Therefore, the susceptibility of the CNS stem cells to CMV is completely different from that of the EC cells, which have the potential to differentiate into many types of cells. It has been reported that a repressor for transcription of the immediate early gene of CMV may be present in the undifferentiated EC cells, and the binding of the repressor to the promoter may not occur in cells that have differentiated in response to retinoic acid (Ghazal and Nelson, 1993; Huang et al, 1996). It seems possible that such a repressor does not exist in the CNS stem cells, although their expression of the MCMV IE gene and the viral replication is delayed to some extent compared with those in MEF (Fig. 2, A and B).

MCMV infection tended to cause a decrease in the number of the CNS stem cells as the infectious titer was increased, probably because of inhibition of pro- 

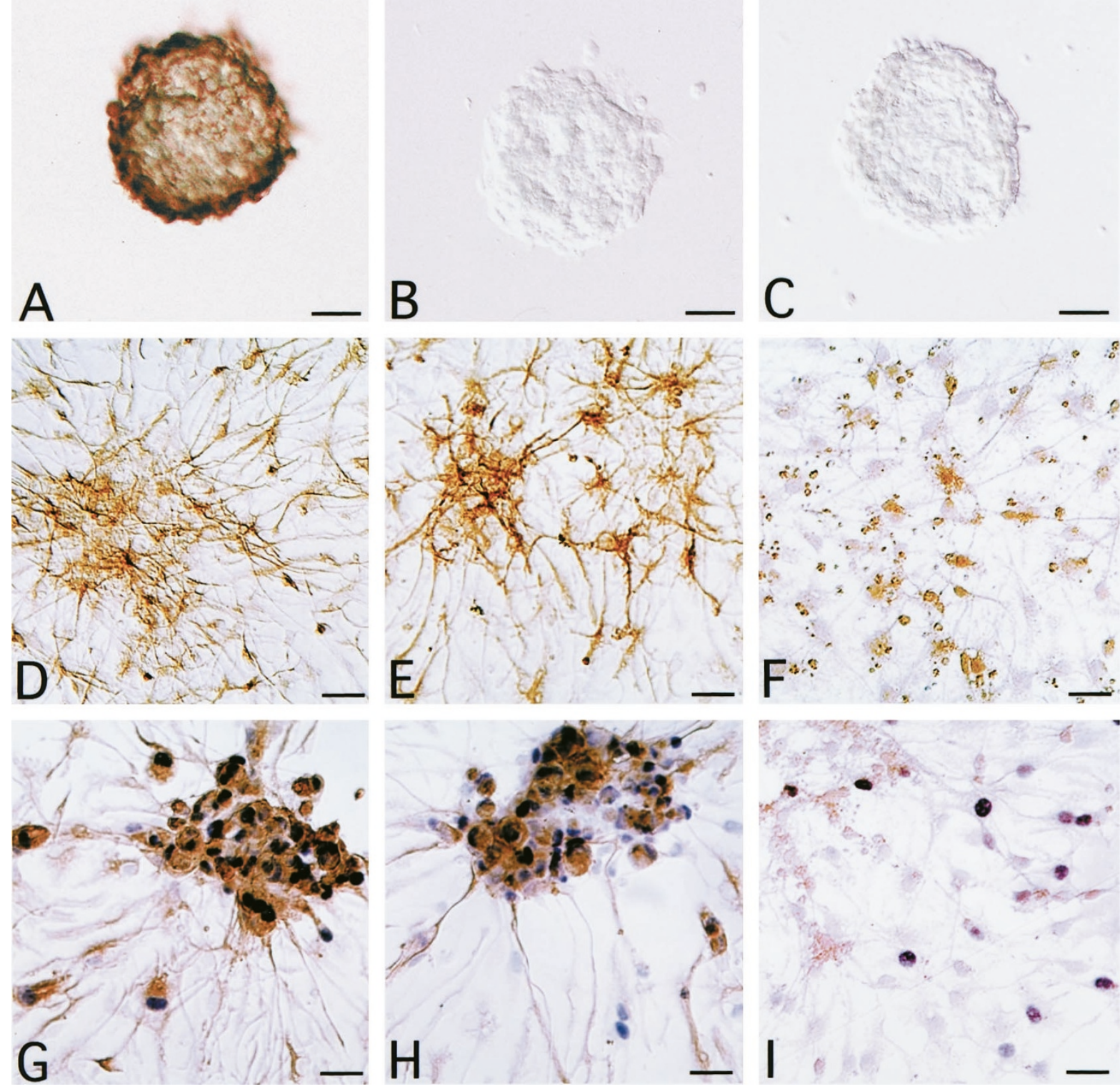

\section{Figure 6.}

Immunocytochemistry of CNS stem cells and the differentiated cells for expression of nestin (A and D), GFAP (B and E), and NF (C and F). A to C, Uninfected spheres. $D$ to $F$, Uninfected cells 36 hours after induction of differentiation as described in the legend for Figure 5. G to I, Double immunocytochemistry for MCMV IE antigen and neural cell markers: nestin (G), GFAP (H), or NF (I) in MCMV-infected cells 36 hours after induction of differentiation (48 hpi). MCMV IE antigen was labeled with the N2 antibody and ALP-conjugated goat anti-rat IgG and colored with Fast blue BB (blue). Neural cell markers were labeled with the rabbit antibodies described in the text and HRP-conjugated swine anti-rabbit IgG and colored with diaminobenzidine (brown). Bar $=20 \mu \mathrm{m}$.

gression through the cell cycle or because of cell loss caused by lytic infection. Decreased incorporation of BrdU into the stem cells expressing the IE antigen indicates that MCMV infection inhibits the DNA synthesis of the CNS stem cells during the IE or early phase of infection. Although induction of cellular DNA synthesis following HCMV infection has long been reported in a variety of cell lines (Albrecht et al, 1993), it was recently reported that HCMV infection inhibits cell cycle progression at the G1/S transition in human fibroblasts (Lu and Shenk, 1996; Dittmer and Mocarski, 1997). In those studies, HCMV infection inhibited the growth of human fibroblasts, and this growth inhibition occurred rapidly after virus infection. Our findings that the cells expressing the IE antigen did not enter $\mathrm{S}$ phase, as detected by BrdU incorporation, may be consistent with those results. It may be noteworthy that the CNS stem cells having features different from those of fibroblasts, which can easily be rendered quiescent, are similarly inhibited in their growth by CMV infection.

By clonogenic assay (Reynolds and Weiss, 1996), generation of spheres of CNS stem cells from single cells was shown to be markedly suppressed by MCMV infection. This dose-dependent suppression in the colony-forming ability of the stem cells by MCMV 


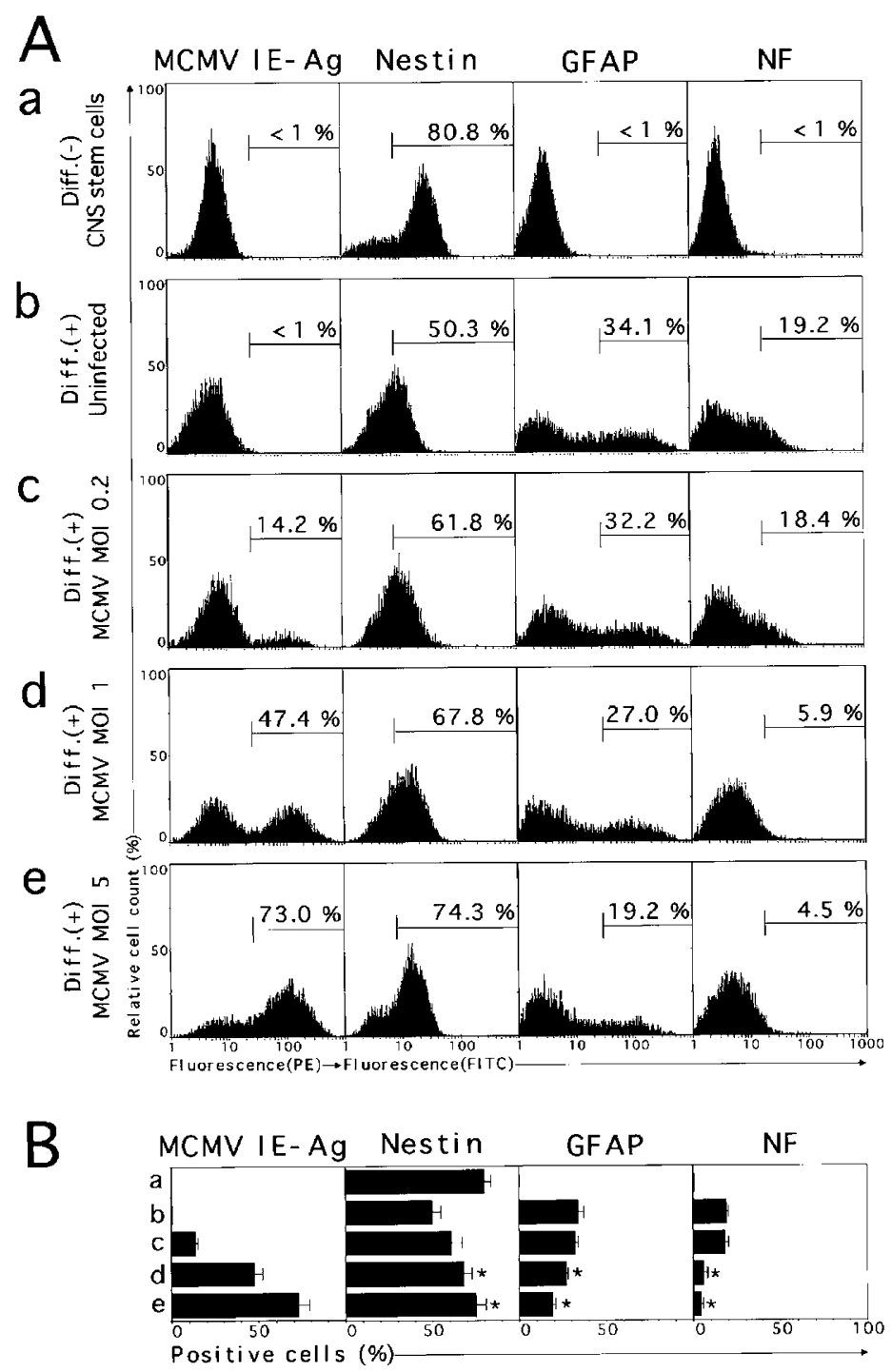

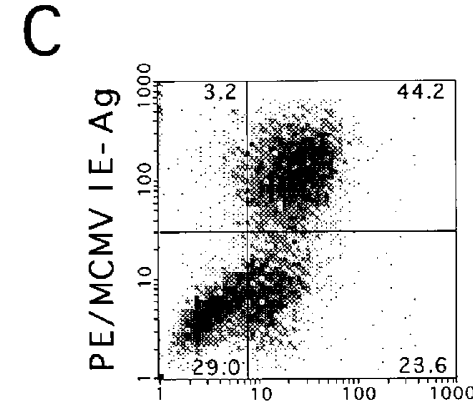

FITC/Nestin

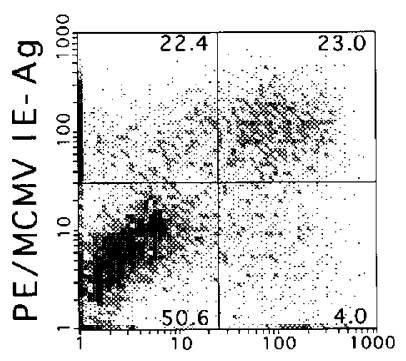

FITC/GFAP

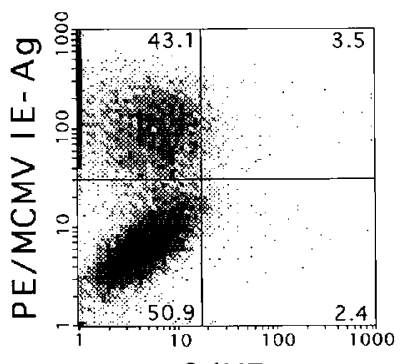

$\mathrm{FITC} / \mathrm{NF}$

Figure 7.

Flow cytometric analysis of the expression of MCMV IE antigen and neural cell markers in uninfected or MCMV-infected cells differentiated from CNS stem cells as described in the legend for Figure 5. The IE antigen or neural cell markers were labeled with PE-conjugated streptavidin or FITC-conjugated antibody, respectively. Ten thousand gated cells were analyzed for each measurement, and the cut-off points were 27.0, 7.6, 28.3, or 15.2, for IE antigen, nestin, GFAP, or NF, respectively. A, Single-color flow cytometry for IE antigen or neural cell markers. Aa, Uninfected stem cells 4 days after passage. Ab, Uninfected cells 36 hours after induction of differentiation. Ac to Ae, MCMV-infected cells 36 hours after induction of differentiation at MOI 0.2 (Ac), MOI 1 (Ad), or MOI 5 ( $\mathrm{Ae}$ ). B, Based on the experiments performed in $\mathrm{A}$, data of three independent experiments were averaged. ${ }^{\star} p<0.05$ compared with uninfected cells by Student's $t$ test. C, Two-color flow cytometry for the expression of the MCMV IE antigen (PE) and neural cell markers (FITC) in the MCMV-infected cells 36 hours after induction of differentiation (48 hpi at MOI 1).

was similar to the suppression observed in hematopoietic progenitor cells infected with HCMV (Maciejewski et al, 1992). As shown in the hematopoietic progenitor cells, a few stem cells still generated spheres even though cells were infected with a high MOI. It is possible that a small population of the CNS stem cells may have resistance or a relatively low permissiveness for MCMV replication, and may be latently infected, like bone marrow progenitor cells (Kondo et al, 1994). However, we have not so far been able to detect the virus genome or virus gene expression in these spheres generated from MCMV-infected stem cells.

The CNS stem cells were induced to differentiate by culturing cells adhering to culture dishes in EGF- depleted, $2 \%$ horse serum-supplemented medium. As differentiation progressed, the intensity of nestin staining decreased, with the appearance of a differentiation marker, as also described by Rajan and McKay (1998). The differentiation of the CNS stem cells seemed to be generally inhibited by MCMV infection, because reduction of nestin-positive cells did not occur. However, MCMV infection inhibited neuronal differentiation more severely than glial differentiation. Several lines of evidence have indicated that neuronal and glial cells are derived from common multipotential precursor cells (Temple, 1989; Reynolds and Weiss, 1992; Vescovi et al, 1993; McKay, 1997). Some regulatory factors have been suggested to have roles in the commitment of the CNS stem cells to form neuron- or 


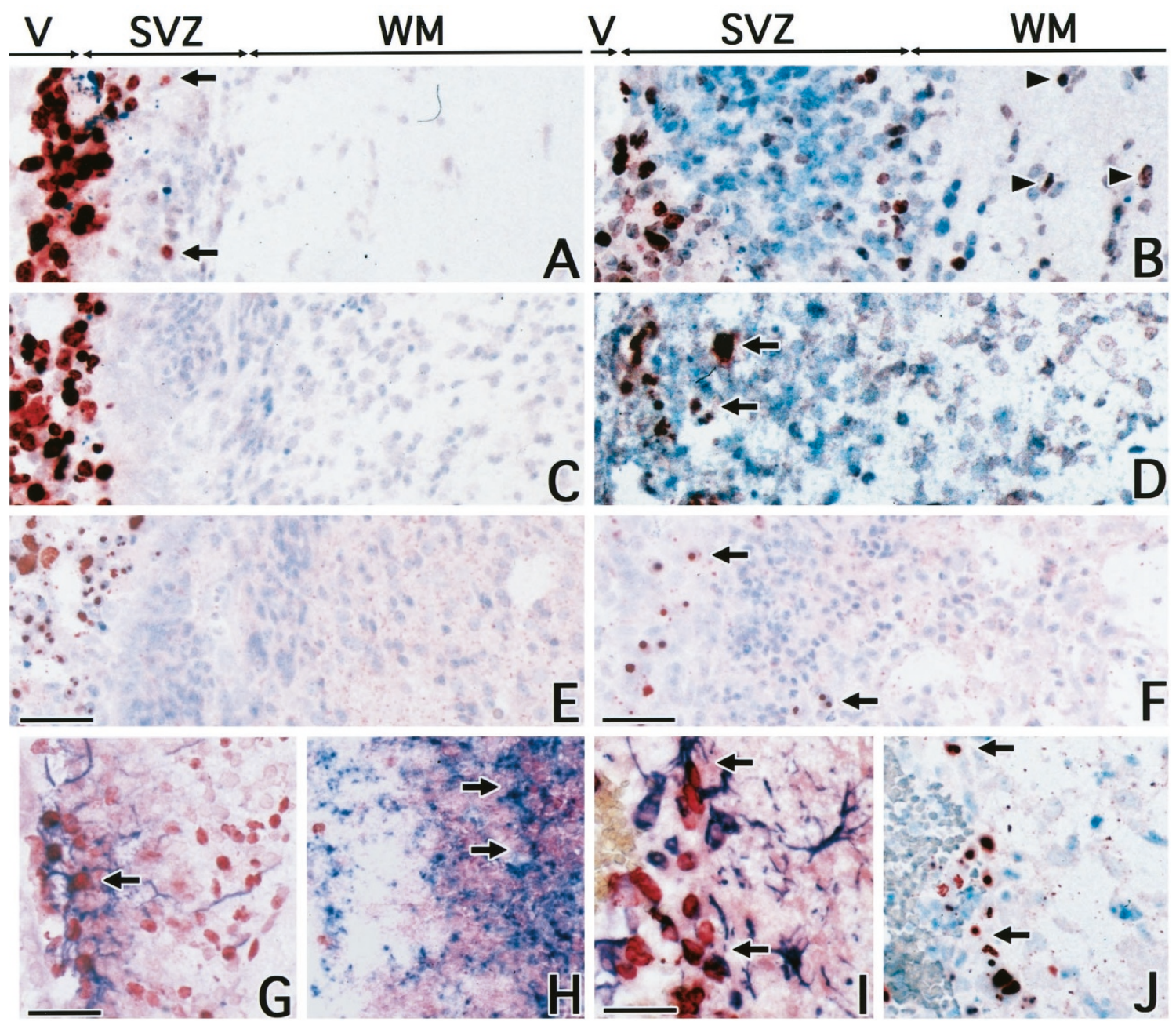

Figure 8 .

Transplantation of MCMV-infected or uninfected CNS stem cells into the neonatal rat brains. Immunohistochemistry of the rat brains 2 days post-transplantation (dpt) $(A, C$, and $E)$ or 6 dpt (B, D, and F) with uninfected ( $A$ and $B$ ) or MCMV-infected CNS stem cells (C to F) which were labeled with BrdU. A to D, BrdU staining. $E$ to F, Viral antigen staining with the antibody specific to the MCMV early antigen (the D5 antibody). C and E, D, and F, The adjacent sections, respectively. Arrows indicate the cells migrated into the SVZ (A, D, and F) and arrowheads indicated cells migrated into the WM (B). G to I, Double immunostaining for BrdU and neural cell markers of the uninfected ( $\mathrm{G}$ and $\mathrm{H}$ ) and MCMV-infected $(I)$ on 6 dpt. G, GFAP (arrow shows double stained cell in inner site of the SVZ). H, $\beta 3$-tubulin (arrows show double-stained cells in outer site of the SVZ). I, BrdU and GFAP (arrows show double-stained cells). J, The adjacent section of $I$, stained with the D5 antibody to the MCMV early antigen (arrows). BrdU and MCMV early antigen were colored with aminoethylcarbazole (red). GFAP and $\beta 3$-tubulin were colored with Fast blue BB (blue). Hematoxylin was used for counter staining (A to F). V, ventricle; SVZ, subventricular zone; WM, white matter. Bar $=50 \mu \mathrm{m}$.

glial-lineages (McKay, 1997). CMV infection may affect the regulatory mechanism by which CNS stem cells undergo neuronal differentiation, but may have less effect on glial differentiation, by down-regulation of some host factors such as major histocompatibility complex class I expression, which is suppressed by CMV infection (Sedmak et al, 1994).

The viral IE antigen was preferentially expressed in the glial precursors expressing GFAP after infection of CNS stem cells with MCMV and stimulation to differentiate. As reported previously in the developing mouse brain infected with MCMV (Shinmura et al 1997a), the IE antigen-positive cells, mainly located in the ventricular and subventricular zones, had characteristics of glial differentiated cells expressing GFAP and nestin. Therefore, glial cells differentiated from CNS stem cells seem to correspond to the glial cells in the ventricular and subventricular zones in the devel- oping brains, and cells both in vitro and in vivo are similarly susceptible to MCMV infection. Because expression of the IE genes is dependent on cellular transcription factors, we examined cell-type-specific activity of the MCMV IE promoter in transgenic mice. It was found that the IE promoter directs astrocytespecific expression in the brain (Aiba-Masago et al, 1999). Interestingly, the promotor activity was found in the cells of the ventricular and subventricular zones at late stages of gestation (R-Y Li, S Baba, I Kosugi, Y Arai, H Kawasaki, Y Shinmura, S Sakakibara, H Okano, and Y Tsutsui, unpublished data).

In the transplantation study, uninfected CNS stem cells were engulfed into the SVZ and migrated into the white matter and the cortex, as described by Brüstle (1999), whereas the engulfed MCMV-infected stem cells were few in number and hardly migrated further. However, these infected stem cells expressed GFAP, 
but not $\beta$ 3-tubulin. These findings suggest that MCMV infection inhibits migration and neuronal differentiation of the CNS stem cells rather than glial differentiation after transplantation, and also suggest that the inhibition of growth and neuronal differentiation of the CNS stem cells by MCMV infection observed in vitro studies may reflect the phenomena occurring in vivo.

Microcephaly and polymicrogyria are the most prominent features of brain abnormalities in congenital CMV infection in humans (Becroft, 1981; Perlman and Argyle, 1992). It is possible that these brain abnormalities may result from cellular events occurring within the ventricular zone in which the stem cells are proliferating, movement of these cells from the ventricular zone, determination to neuronal or glial lineages, and then migration of these cells and selective removal of the neuronal cells by apoptosis (Crino and Eberwine, 1997). We have already reported some evidence that MCMV infection may disturb neuronal migration (Shinmura et al, 1997b) and induce apoptosis in the developing mouse brain (Kosugi et al, 1998), and that the susceptibility of the glial cells to MCMV may be greater than that of neuronal cells (Shinmura et al, 1997a). In this report, we showed that the CNS stem cells are permissive for MCMV infection, and that the MCMV infection inhibits the growth of the stem cells. In addition, we suggest that CMV infection affects the differentiation of neuronal cells from the CNS stem cells or their precursor cells.

Because the ventricular and subventricular zones are thought to be primary targets in the brain during congenital CMV infection, the infectious dynamics of the CNS stem cells may provide a model system for the pathogenesis of the brain abnormalities in congenital CMV infection in humans.

\section{Materials and Methods}

\section{Virus and Cell Culture}

The Smith strain of MCMV, which had been passaged in MEF cells, was provided by Dr. Y. Minamishima (Miyazaki, Japan; Ebihara and Minamishima, 1984). MEF cells were prepared from 12.5-day-old embryos of BALB/c mice (SLC Japan, Hamamatsu, Japan), and were grown in DMEM containing penicillin (100 units/ $\mathrm{ml})$, streptomycin $(50 \mu \mathrm{g} / \mathrm{ml})$, and $10 \%$ fetal bovine serum (FBS), as described previously (Tsutsui and Naruse, 1987). The titer of the virus stock was $4 \times 10^{8}$ $\mathrm{PFU} / \mathrm{ml}$. Ultraviolet light (UV)-inactivated MCMV was used for control experiments. The UV-irradiated MCMV did not replicate or produce any detectable level of the IE protein.

\section{Murine CNS Stem Cell Culture and Virus Infection}

Murine EGF-responsive CNS stem cells were prepared from the cerebral hemispheres of ICR mouse embryos at day 14 of gestation. After mechanical dissociation, the cell suspensions were grown in the defined serum-free medium containing EGF (purified from mouse submaxillary glands; Becton Dickinson,
Bedford, MA) at $20 \mathrm{ng} / \mathrm{ml}$ as described by Reynolds and Weiss (1996). The cultures were passaged every 4 to 5 days by centrifugation of spheres, followed by dissociation to single-cell suspensions. Spheres of the CNS stem cells passaged 5 to 10 times were used for the experiments.

The dissociated stem cells in suspension or MEF were infected with MCMV at different $\mathrm{MOI}$, washed by centrifugation, and cultured with fresh medium. At different times after infection, three samples were collected and counted by trypan blue exclusion. The viral titers were measured in aliquots of the cells by a plaque assay using MEF. Other cell aliquots were processed for detection of MCMV antigen by immunofluorescence and flow cytometry.

\section{Analysis of DNA Synthesis of CNS Stem Cells}

Two days after passage, the CNS stem cells were infected with MCMV at MOI 2 or 10, washed by centrifugation, then cultured with fresh medium. At 15 hpi, when viral DNA had not yet begun to accumulate, the cells were incubated with $15 \mu \mathrm{M}$ BrdU for 30 minutes, and then trypsinized and fixed with $4 \%$ paraformaldehyde. The cells were rinsed with T-PBS (PBS containing $0.2 \%$ Triton-X-100 and 5\% FBS), reacted with rat anti-MCMV IE antigen in T-PBS, and then washed and stained with biotin-conjugated goat anti-rat IgG (Pharmingen, San Diego, CA) and phycoerythrin (PE)-conjugated streptavidin (Gibco BRL, Gaithersburg, MD). After washing, the cells were fixed again, treated with $2 \mathrm{~N} \mathrm{HCl}$ for 10 minutes at room temperature, stained with mouse anti-BrdU (DACO, Kyoto, Japan) and fluorescein isothiocyanate (FITC)conjugated goat $\mathrm{F}\left(\mathrm{ab} \mathrm{b}^{\prime}\right)_{2}$ anti-mouse $\mathrm{F}\left(\mathrm{ab} \mathrm{b}^{\prime}\right)_{2}$ (Cappel, West Chester, PA), and subjected to flow cytometry.

\section{Clonogenic Assay}

The spheres of CNS stem cells were mechanically dissociated, verified to be completely dispersed by examination using a hemocytometer, and infected with MCMV at different $\mathrm{MOI}(0.1,1$, and 10$)$ for 1 hour. After washing, they were dissociated and again verified to be dispersed, and then diluted and plated into 96-well plates at a density of 6 cells per well. Because of the decrease in cell number after the mechanical dissociation as described by Reynolds and Weiss (1996), the plating conditions had been defined in preliminary experiments so that a single live cell could be plated per well in more than $80 \%$ of the wells. Three independent experiments were performed and the number of spheres was counted at $7 \mathrm{dpi}$.

\section{Induction of Differentiation of CNS Stem Cells}

Induction of differentiation of the CNS stem cells was performed as described by Reynolds and Weiss (1996). The stem cells of spheres cultured for 4 days after passage were infected with MCMV at three different $\mathrm{MOI}(0.2,1$, and 5). At $12 \mathrm{hpi}$, the spheres were washed by centrifugation, plated onto glass coverslips or 6-well plates that had been coated with 
$100 \mu \mathrm{g} / \mathrm{ml}$ poly-D-lysine (Sigma, St. Louis, MO) and 20 $\mu \mathrm{g} / \mathrm{ml}$ laminin (Upstate Biotechnology, Lake Placid, $\mathrm{NY}$ ), at a density of $5 \times 10^{4} \mathrm{cells} / \mathrm{cm}^{2}$ or $2.5 \times 10^{6}$ cells per plate. The spheres were induced to differentiate in medium lacking EGF but supplemented with $2 \%$ heat-inactivated horse serum (Gibco). At 36 hours after differentiation (48 hpi), the cells cultured on coverslips were fixed with $4 \%$ paraformaldehyde (PFA) and analyzed by immunocytochemistry; the cells cultured in 6-well plates were collected by treatment with 4 units/ml papain (Sigma) and $1 \mathrm{~mm}$ EDTA, fixed with $4 \%$ PFA, and analyzed by flow cytometry. Three independent experiments were performed, and the average number of live cells recovered in both uninfected and MCMV-infected cultures was $2.4 \pm$ $0.2 \times 10^{6}$ cells per plate.

\section{Immunocytochemistry}

Immunohistochemical double staining was performed as described previously (Kosugi et al, 1998). The fixed cells on cover slips were stained with a rat anti-MCMV IE antigen and a rabbit polyclonal antibody to a neuroepithelial cell marker; anti-nestin (provided by Dr. H. Kitani, National Institute for Animal Health, Tukuba, Japan; Tomooka et al, 1993), anti-bovine GFAP (DACO, Japan), or anti-mouse 200 kD NF (Sigma), incubated with alkaline phosphatase (ALP)-conjugated goat anti-rat lgG (Pharmingen) and horseradish peroxidase (HRP)conjugated swine anti-rabbit IgG (DACO Japan), and then colored with Fast-blue BB (blue) and 3,3'diaminobenzidine-4 $\mathrm{HCl}$ (brown), respectively. The stained cells were observed with Nomarski interferencecontrast microscopy (Olympus, Tokyo).

\section{Flow Cytometry}

For flow cytometry, spheres of the CNS stem cells were treated with $0.05 \%$ trypsin and $1 \mathrm{~mm}$ EDTA and differentiated precursor cells were dissociated with 4 units/ml papain and $1 \mathrm{~mm}$ EDTA, and then fixed with $4 \%$ paraformaldehyde. The cells were reacted in suspension with primary antibodies as described above, and then washed with T-PBS. For the secondary labeling, biotin-conjugated goat anti-rat IgG and PEconjugated streptavidin, or FITC-conjugated goat $F\left(a b^{\prime}\right)_{2}$ anti-rabbit $F\left(a b^{\prime}\right)_{2}$ (Cappel) were used for the detection of the MCMV antigen or cell markers, respectively. The stained cells were analyzed by flow cytometry using an EPICS profile analyzer (Coulter, Miami, FL), with $1 \times 10^{4}$ gated cells recorded for each analysis. Absence of cross-reactivity of all of the secondary antibodies was verified.

\section{Transplantation of the CNS Stem Cells into Neonatal Rat Brain}

Transplantation of the CNS stem cells was performed basically as described by Svendsen et al (1996). The CNS stem cells cultured for 4 days were labeled with 0.5 $\mu \mathrm{M}$ BrdU for 24 hours, then infected with MCMV at MOI 1 for 3 hours, and washed with the growth medium with EGF three times by centrifuge ( $\times 500$ rpm for 5 minutes).
After dissociation in the presence of $0.001 \%$ deoxyribonuclease (Sigma), the stem cells were pelleted and suspended in the growth medium. Newborn rats (within 24 hours after birth) were transplanted with MCMVinfected or uninfected cells $\left(2 \times 10^{6}\right.$ cells $\left./ 5 \mu l\right)$ into the right cerebral hemisphere by a $10-\mu$ l Hamilton syringe with a 27-gauge needle from a middle point between the ear and eye. After transplantation, the neonatal rats were killed 2 and 6 days after transplantation. After fixation in $4 \%$ PFA for 2 days at $4^{\circ} \mathrm{C}$, the brains were immersed in $20 \%$ sucrose in PBS, frozen in n-hexane at $-70^{\circ} \mathrm{C}$, and sectioned by the cryostat. The coronal sections were subjected to immunohistochemistry, as described above. For detection of neuronal differentiation, anti- $\beta$ 3-tubulin antibody (Sigma) was used. The D5 antibody specific to the early nuclear antigen of MCMV (Tsutsui and Naruse, 1987) was used for detection of the infected cells.

\section{Acknowledgements}

We thank Mr. Kiyoshi Shibata, Research Equipment Center of Hamamatsu University School of Medicine, for his technical assistance with flow cytometry.

\section{References}

Aiba-Masago S, Satoshi B, Li RY, Shinmura Y, Kosugi I, Arai Y, Nishimura M, and Tsutsui Y (1999). Murine cytomegalovirus immediate-early promoter directs astrocyte-specific expression in transgenic mice. Am J Pathol 154:735-743.

Albrecht T, Boldogh I, Fons MP, and Valyi-Nagy T (1993). Activation of proto-oncogenes and cell activation signals in the initiation and progression of human cytomegalovirus infection. In: Becker $\mathrm{Y}$, Darai $\mathrm{G}$, and Huang $\mathrm{E}$, editors. Frontiers of virology 2: Molecular aspects of human cytomegalovirus diseases. New York: Springer-Verlag, 384-411.

Bale JF Jr (1984). Human cytomegalovirus infection and disorders of the nervous system. Arch Neurol 41:310-320.

Becroft DMO (1981). Prenatal cytomegalovirus infection: Epidemiology, pathology, pathogenesis. In: Rosenberg HS and Bernstein J, editors. Perspectives in pediatric pathology. New York: Masson, 203-241.

Brüstle O (1999). Building brains: Neural chimeras in the study of nervous system development and repair. Brain Pathol 9:527-545.

Chenn A and McConnell SK (1995). Cleavage orientation and the asymmetric inheritance of Notch-1 immunoreactivity in mammalian neurogenesis. Cell 82:631-641.

Crino PB and Eberwine J (1997). Cellular and molecular basis of cerebral dysgenesis. J Neurosci Res 50:907-916.

Davis AA and Temple S (1994). A self-renewing multipotential stem cells in embryonic rat cerebral cortex. Nature 372:263266.

Demmler GJ (1991). Infectious Disease Society of American and Centers of Disease Control: Summary of a workshop on surveillance for congenital cytomegalovirus disease. Rev Infect Dis 13:315-329.

Dittmer D and Mocarski ES (1997). Human cytomegalovirus infection inhibits G1/S transition. J Virol 71:1629-1634. 
Dutko FJ and Oldstone MBA (1981). Cytomegalovirus causes a latent infection in undifferentiated cells and is activated by induction of cell differentiation. J Exp Med 154:1636-1651.

Ebihara K and Minamishima $Y$ (1984). Protective effect of biological response modifiers on murine cytomegalovirus infection. J Virol 51:117-122.

Ghazal P and Nelson JA (1993). Transcription factors and viral regulatory proteins as potential mediators of human cytomegalovirus pathogenesis. In: Becker Y, Darai G, and Huang E, editors. Frontiers of virology 2: Molecular aspects of human cytomegalovirus diseases. New York: Springer-Verlag, 360-383.

Gonczol E, Andrews PW, and Plotokin SA (1984). Cytomegalovirus replicates in differentiated but not in undifferentiated human embryonal carcinoma cells. Science 224:159-161.

Gonczol E, Andrews PW, and Plotokin SA (1985). Cytomegalovirus infection of human teratocarcinoma cells in culture. J Gen Virol 66:509-515.

Ho M (1991). Congenital and perinatal human cytomegalovirus infection. In: Cytomegalovirus: Biology and infection. New York: Plenum, 205-277.

Huang TH, Oka T, Asai T, Okada T, Merrills BW, Gertson PN, Whitson $\mathrm{RH}$, and Itakura K (1996). Repression by a differentiation-specific factor of the human cytomegalovirus enhancer. Nucleic Acids Res 24:1695-1701.

Kashiwai A, Kawamura N, Kadota C, and Tsutsui Y (1992). Susceptibility of mouse embryo to murine cytomegalovirus infection in early and mid-gestation stages. Arch Virol 127: 37-48.

Kondo K, Kaneshima H, and Mocarski ES (1994). Human cytomegalovirus latent infection of granulocyte-macrophage progenitors. Proc Natl Acad Sci USA 91:11879-11883.

Kosugi I, Shinmura Y, Li RY, Aiba-Masago S, Baba S, Miura $K$, and Tsutsui $Y$ (1998). Murine cytomegalovirus induces apoptosis in non-infected cells of the developing mouse brain and blocks apoptosis in primary neuronal culture. Acta Neuropathol 96:239-247.

Lu M and Shenk T (1996). Human cytomegalovirus infection inhibits cell cycle progression at multiple points, including the transition from G1 to S. J Virol 70:8850-8857.

Maciejewski JP, Bruening EE, Donahue RE, Mocarski ES, Young NS, and St Jeor SC (1992). Infection of hematopoietic progenitor cells by human cytomegalovirus. Blood 80:170-178.

McKay R (1997). Stem cells in the central nervous system. Science 276:66-71.

Perlman JM and Argyle C (1992). Lethal cytomegalovirus infection in preterm infants: Clinical, radiological, and neuropathological findings. Ann Neurol 31:64-68.

Potten CS and Loeffler M (1990). Stem cells: Attributes, cycles, spirals, pitfalls and uncertainties. Lessons for and from the crypt. Development 110:1001-1020.

Rajan P and McKay DG (1998). Multiple routes to astrocytic differentiation in the CNS. J Neurosci 18:3620-3629.

Reynolds BA and Weiss S (1992). Generation of neurons and astrocytes from isolated cells of the adult mammalian central nervous system. Science 255:1707-1710.

Reynolds BA and Weiss S (1996). Clonal and population analyses demonstrate that an EGF-responsive mammalian embryonic CNS precursor is a stem cell. Dev Biol 175:1-13.
Sedmak DD, Guglielmo AM, Knight DA, Birmingham DJ, Huang EH, and Waldman WJ (1994). Cytomegalovirus inhibits major histocompatibility class II expression on infected endothelial cells. Am J Pathol 144:683-692.

Shinmura Y, Aiba-Masago S, Kosugi I, Li RY, Baba S, and Tsutsui $Y$ (1997a). Differential expression of the immediateearly antigens in neuronal and glial cells of developing mouse brains infected with murine cytomegalovirus. Am J Pathol 151:1331-1340.

Shinmura Y, Kosugi I, Aiba-Masago S, Baba S, Li RY, and Tsutsui $Y$ (1997b). Disordered migration and loss of virusinfected neuronal cells in developing mouse brains infected with murine cytomegalovirus. Acta Neuropathol 93:551-557.

Stagno S, Pass RF, Cloud G, Britt WJ, Henderson RE, Walton PD, Veren DA, Page F, and Alford CA (1986). Primary cytomegalovirus infection in pregnancy: Incidence, transmission to fetus, and clinical course. JAMA 256:1904-1908.

Stemple DL and Mahanthappa NK (1997). Neural stem cells are blasting off. Neuron 18:1-4.

Svendsen CN, Clarke DJ, Rosser AE, and Dunnett SB (1996). Survival and differentiation of rat and human epidermal growth factor-responsive precursor cells following grafting into the lesioned adult central nervous system. Exp Neurol 137:376-388.

Temple S (1989). Division and differentiation of isolated CNS blast cells in microculture. Nature 340:471-473.

Tomooka Y, Kitani H, Jing N, Matsushima M, and Sakakura T (1993). Reconstruction of neural tube-like structure in vitro from primary neural precursor cells. Proc Natl Acad Sci USA 90:9683-9687.

Tsutsui $Y$ (1995). Developmental disorders of the mouse brain induced by murine cytomegalovirus: Animal models for congenital cytomegalovirus infection. Pathol Int 45:91-102.

Tsutsui Y, Kashiwai A, Kawamura N, and Kadota C (1993). Microphthalmia and cerebral atrophy induced in mouse embryos by infection with murine cytomegalovirus in midgestation. Am J Pathol 143:804-813.

Tsutsui $Y$ and Naruse I (1987). Murine cytomegalovirus infection of cultured mouse embryos. Am J Pathol 127:262270.

Tucker RP (1990). The roles of microtubule-associated proteins in brain morphogenesis: A review. Brain Res Rev 15:101-120.

van den Pol AN, Mocarski E, Saederup N, Vieira J, and Meier TJ (1999). Cytomegalovirus cell tropism, replication, and gene transfer in brain. J Neurosci 19:10948-10965.

Vescovi AL, Parati EA, Gritti A, Poulin P, Ferrario M, Wanke E, Frolichsthal-Schoeller P, Cova L, Arcellana-Panlilio M, Colombo A, and Galli R (1999). Isolation and cloning of multipotential stem cells from the embryonic human CNS and establishment of transplantable human neural stem cell lines by epigenetic stimulation. Exp Neurol 156:71-83.

Vescovi AL, Reynolds BA, Fraser DD, and Weiss S (1993). bFGF regulates the proliferative fate of unipotent (neuronal) and bipotent (neuronal/astroglial) EGF-generated CNS progenitor cells. Neuron 11:951-966.

Weller TH (1971). The cytomegalovirus: Ubiquitous agents with protean clinical manifestations. N Engl J Med 285:203214. 